\title{
A New Proof of Williams' Decomposition of the Bessel Process of Dimension Three with a Look at Last-Hitting Times
}

\author{
F. Thomas Bruss Marc Yor
}

\begin{abstract}
In this note we propose a concise proof of David Williams' decomposition of the Bessel Process of dimension 3 (BES(3)), starting from $r>0$ at its ultimate minimum. An ultimate minimum of a stochastic process may be seen as a state of a process at a last hitting time. This discussion is strongly motivated by our interest in properties of last hitting times in general, and here specifically, directly linked with the reading guide of Nikeghbali and Platen (2013).
\end{abstract}

\section{Introduction}

Clearly, to decide whether a stochastic process hits a certain set for the last time depends on what will happen in the future. Therefore last-hitting times are usually not measurable with respect to the natural filtration of the process and thus among the difficult random times of a stochastic process. Stopping times, on the contrary, do have this property by definition, and we know quite an impressive collection of Theorems and tools for stopping times.

As Chung (see citation of Nikeghbali and Platen (2013)) among others conclude, last hitting times must be avoided at all costs.

Received by the editors in January 2014 - In revised form in January 2015.

Communicated by S. Caenepeel.

2010 Mathematics Subject Classification : 60 H 30; secondary 60 G 40.

Key words and phrases: Brownian motion, Ornstein-Uhlenbeck process, stopping times, measurability, 1/e-law of best choice, last arrival problem, proportional increment process, compassionate-use clinical trials. 
This is one way to see things, but, often enough, reality looks somewhat different. Indeed, ironically, many interesting problems in the theory of optimal stopping require us to deal with last hitting times, and not with stopping times. And so, the attitude has changed, and the work of Jeulin (1980) and others had quite an influence on this development. In their recent paper, Nikeghbali and Platen cite several interesting examples from the domain of Mathematical Finance, and we look at one of them under a different angle. We would also like to slightly broaden the horizon of Mathematical Finance by looking at a few other examples. But first to the main topic of this article, that is, to David Williams' theorem of decomposition.

\section{The BES(3)-process and Williams' Theorem}

(1.1) In their survey about last passage times, Nikeghbali and Platen (2013) illustrate some of their formulae with the following example:

Let $\left(R_{t}\right)_{t \geq 0}$ be a 3-dimensional Bessel process (BES(3)-process) on $\mathbb{R}_{+}$starting from $r>0$. Denote by $\left(\mathcal{F}_{t}\right)_{t \geq 0}$ its natural filtration, and let $I_{t}$ denote the current infimum of the process $(R)$ at time $t$, that is,

$$
I_{t}=\inf _{0 \leq s \leq t} R_{s}
$$

The following results can be found in Nikeghbali and Platen around Corollary 4.10:

(a) $I_{\infty}$ follows the same distribution as the random variable $r U$, where $U$ is uniform on $[0,1]$.

(b) The Azéma-supermartingale associated with the random time $g$ at which the process $\left(R_{t}\right)_{t \geq 0}$ reaches $I_{\infty}$ is given by

$$
Z_{t} \equiv P\left(g>t \mid \mathcal{F}_{t}\right)=\frac{I_{t}}{R_{t}}
$$

(c) The Laplace transform of the law of $g$ is

$$
\mathrm{E}\left(e^{-\lambda g}\right)=\frac{1}{\sqrt{2 \lambda} r}\left(1-e^{-\sqrt{2 \lambda} r}\right)
$$

(d) The density of $g$ denoted by $p(t)$ equals

$$
p(t)=\frac{1}{\sqrt{2 \pi t} r}\left(1-e^{-\left(r^{2} / 2 t\right)}\right) .
$$

Our aim is now to show Williams' decomposition of a BES(3)-process at its ultimate minimum, and how this decomposition is closely connected with (a)-(b)-(c)-(d). 
(1.2) Recall that if $\left(B_{t}\right)_{t \geq 0}$ is a Brownian motion starting from 0 and $a$ is a real constant, then the law of the first hitting time of $a$ by $\left(B_{t}\right)$, denoted by $T_{a}^{(B)}$ is given by

$$
P\left(T_{a}^{(B)} \in d t\right)=\frac{d t}{\sqrt{2 \pi t^{3}}}|a| \exp \left(-\frac{a^{2}}{2 t}\right) .
$$

This well-known fact allows us to rewrite the statements (c) and (d) above as

$$
g \stackrel{\mathcal{L}}{=} T_{r U}^{(B)}
$$

where $U$ is independent of $(B)$ and uniform on $[0,1]$, and where $\underline{\mathcal{L}}$ denotes identity in law. This can be verified using (c) and (d). In fact, (2) may be understood via the classical decomposition of the process $(R)$ before and after time $g$, due to Williams (1974).

The essence of the result is displayed in Figure 1.

\section{Williams' decomposition of $(R)$, before and after $g$, via pro- gressive enlargement}

(2.1) Figure 1 below displays a 'finite-horizon' version of the decomposition of a BES(3)-process.

Note that this figure is nothing else but a (simulated finite-horizon) version of the Figure 5 in Revuz-Yor (1999) (see Proposition 3.10 and Theorem 3.11 in Ch. 6, Sect. 3) where the BES(3)-process is considered starting from level $c:=r$.

(2.2) We now state precisely Williams' Decomposition Theorem before and after time $g$.

Theorem 1 (Williams (1974))

Consider the following three independent random objects:

(i) a Brownian motion $\left(B_{t}^{\prime}\right)_{t \geq 0}$ with $B_{0}^{\prime}=r>0$;

(ii) a uniform random variable $U$ on $[0,1]$;

(iii) a $\operatorname{BES}(3)$-process $\left(\tilde{R}_{t}\right)_{t \geq 0}$ with $\tilde{R}_{0}=0$;

Then the process $(R)$ defined by

$$
R_{t}= \begin{cases}B_{t}^{\prime} & , \text { if } t \leq g \\ r U+\tilde{R}_{t-g} & , \text { if } t \geq g\end{cases}
$$

with $g=\inf \left\{u \geq 0: B_{u}^{\prime}=r U\right\}$ is a BES(3)-process starting from $r>0$.

We note that the pre- $g$-Brownian-motion found in (3) explains the result (2). Indeed, if $B_{t}^{\prime}=r-B_{t}^{(0)}$ then

$$
g=\inf \left\{u \geq 0: B_{u}^{(0)}=r(1-U)\right\}
$$

which implies (2). 


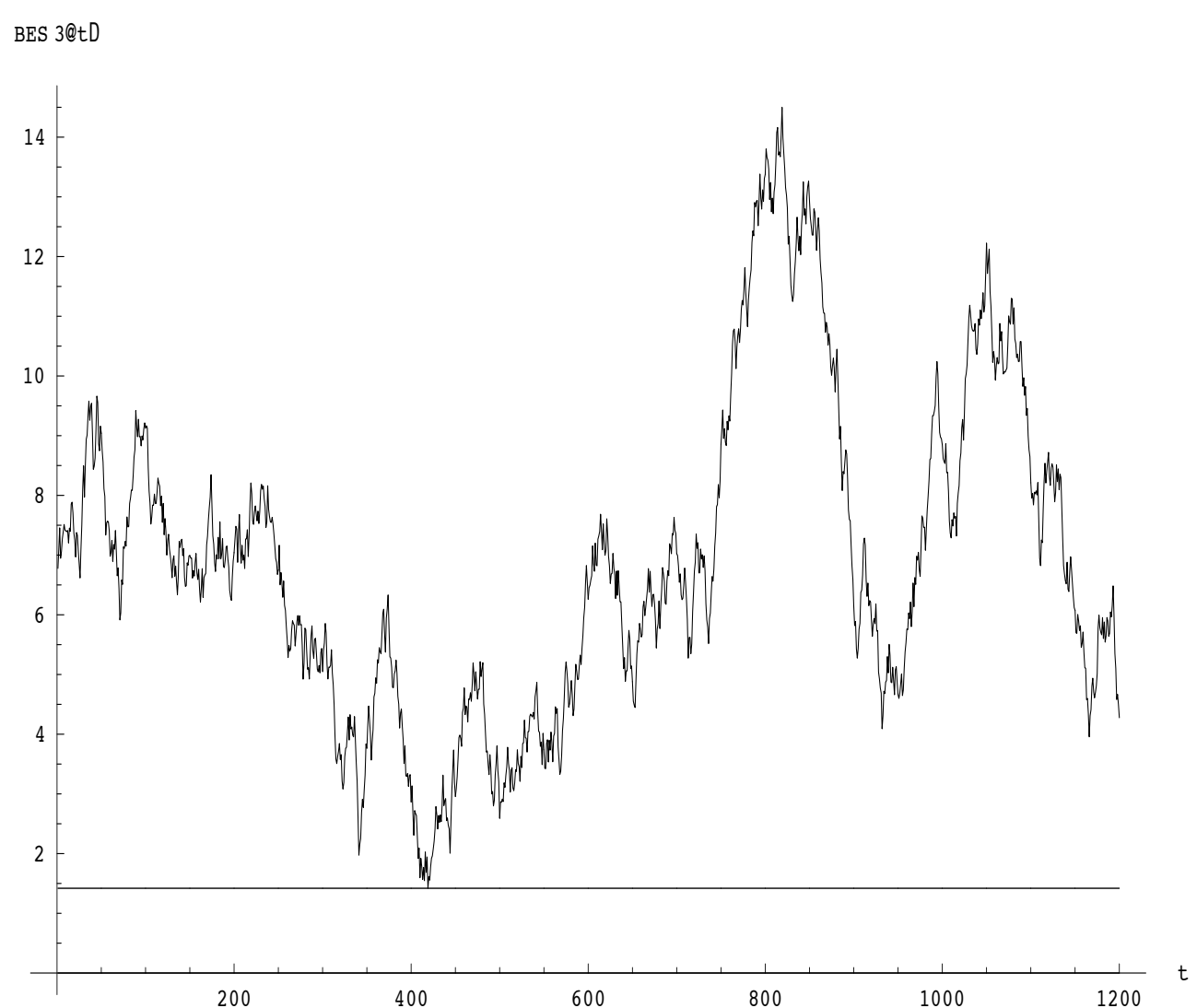

Figure 1: This graph presents an approximation of a BES(3)-process based on three independent simulations of $U[-1 / 2,1 / 2]$-random walks $S_{k}^{x}, S_{k}^{y}, S_{k}^{z}$, where $k$ runs from 1 to 1200 . The starting point is chosen $S_{0}^{x}=4, S_{0}^{y}=4, S_{0}^{z}=2$, so that the starting level of the simulated process is $B_{0}=r=6$. The minimum height is indicated by the supporting horizontal line at level 1.41963. This level is a simulated $I_{1200}$ and not $I_{\infty}$, of course. The latter is unconditionally the random variable $U\left[0, B_{0}\right]=U[0,6]$.

2.3 We now proceed to the proof of the Theorem via the enlargement formula which describes the additive decomposition of the BES(3)-process $\left(R_{t}\right)$ in the filtration $\left(\mathcal{F}_{t}^{g}\right)$ ) containing the filtration $\left(\mathcal{F}_{t}\right)$, and making $g$ a stopping time.

Firstly, we have

$$
R_{t}=r+B_{t}+\int_{0}^{t} \frac{d s}{R_{s}}
$$

where $\left(B_{t}\right)$ is a Brownian motion with respect to the filtration $\left(\mathcal{F}_{t}\right)$.

Secondly, the enlargement formula (see e.g. Jeulin (1980)) yields

$$
r+B_{t}=B_{t}^{\prime}+\int_{0}^{g \wedge t} \frac{d<B, Z>_{u}}{Z_{u}}+\int_{g}^{t} \frac{d<B, 1-Z>_{u}}{1-Z_{u}}
$$

with $\left(B_{t}^{\prime}\right)$ being a Brownian motion with respect to $\left(\mathcal{F}_{t}^{g}\right)$. 
Thirdly, we deduce from (b) the two identities

$$
\frac{d<B, Z>_{u}}{Z_{u}}=-\frac{d u}{R_{u}}, \text { for } u \leq g
$$

and

$$
\frac{d<B, 1-Z>_{u}}{1-Z_{u}}=\frac{I_{\infty} d u}{R_{u}\left(R_{u}-I_{\infty}\right)}, \text { for } u>g .
$$

These two identities imply (using (4) and (6)), and also

$$
\frac{1}{R_{u}}+\frac{I_{\infty}}{R_{u}\left(R_{u}-I_{\infty}\right)}=\frac{1}{R_{u}-I_{\infty}}
$$

the form of the pre- $g$-process, and the form of the post- $g$-process.

Finally, for the proof of (3) to be complete, it remains to prove that the process $\left(B^{\prime}\right)$ is independent of the random variable $I_{\infty} \stackrel{\mathcal{L}}{=} r U$, or more precisely, that, given $I_{\infty}=a$, the pre- $g$-process is just the process $\left(B_{u}^{\prime}\right)_{u \leq T_{a}^{\prime}}$ with obvious notation. This is asserted in the following proposition:

Proposition: Let $\left(\Phi_{u}\right)_{u \geq 0}$ be a non-negative predictable process on path-space. Further, let $P_{r}$ denote the law of the process $(R)$ starting from $r$ and let $P_{r}^{\prime}$ denote the law of the Brownian motion $\left(B^{\prime}\right)$ starting from $r$. Then, for $a<r$,

$$
\begin{aligned}
\mathrm{E}_{r}\left[\Phi_{g} \mid I_{\infty}=a\right] & =\mathrm{E}_{r}\left[\Phi_{T_{a}} \mid T_{a}<\infty\right] \\
& \equiv \mathrm{E}_{r}\left[\Phi_{T_{a}} \mid I_{\infty}<a\right] \\
& =\mathrm{E}_{r}^{\prime}\left[\Phi\left(B_{u}^{\prime} ; u \leq T_{a}^{\prime}\right)\right] .
\end{aligned}
$$

Proof: The equality between the RHS of (8.1) and (8.3) follows, as we will show, from Doob's absolute continuity relationship, namely

$$
P_{r} / \mathcal{F}_{t}=\left(\frac{X_{t \wedge T_{0}}}{r}\right) P_{r}^{\prime} / \mathcal{F}_{t}
$$

on the canonical path-space $C([0, \infty], \mathbb{R})$, where $\left(X_{t}\right)$ denotes the coordinate process on path-space. Indeed, this equality may be extended when replacing the time $t$ by a stopping time. Restricting $\mathcal{F}_{T_{a}}$ on the set $\left\{T_{a}<\infty\right\}$ we get then in particular

$$
P_{r} /\left(\mathcal{F}_{T_{a}} \cap\left\{T_{a}<\infty\right\}\right)=\left(\frac{a}{r}\right) P_{r}^{\prime} / \mathcal{F}_{T_{a}}, 0<a<r,
$$

which yields the desired result.

Identity (8.2) is obvious, since the equality $\left\{T_{a}<\infty\right\}=\left\{I_{\infty}<a\right\}$ holds $P_{r^{-}}$ almost surely.

The proof of the equality (8.1) is slightly more subtle. We start with the identity

$$
\mathrm{E}\left[\mathbf{1}_{\{g \leq t\}} \varphi\left(I_{\infty}\right)\right]=E\left[\left(1-Z_{t}\right) \int_{0}^{t} \varphi\left(I_{s}\right) d\left(1-Z_{s}\right)\right]
$$


which holds for any Borel-measurable function $\varphi:\left[0, \infty\left[\rightarrow \mathbb{R}_{+}\right.\right.$. To see this, note that

$$
\mathrm{E}\left[\mathbf{1}_{\{g \leq t\}} \varphi\left(I_{t}\right)\right]=\mathrm{E}\left[\left(1-Z_{t}\right) \varphi\left(I_{t}\right)\right] .
$$

Assuming $\varphi \in \mathcal{C}^{1}$ the latter becomes by partial integration

$$
\mathrm{E}\left[\int_{0}^{t} \varphi^{\prime}\left(I_{s}\right) d I_{s}\left(1-Z_{s}\right)\right]+\mathrm{E}\left[\int_{0}^{t} \varphi\left(I_{S}\right) d\left(1-Z_{s}\right)\right] .
$$

We note that the expectation involving $\varphi^{\prime}$ vanishes, since $1-Z_{S}$ vanishes $d I_{S}$ almost everywhere. Thus a monotone class argument implies that (9) holds for every non-negative Borel-measurable function $\varphi$.

Next, from the additive decomposition of $\left(1-Z_{s}\right)$, we obtain

$$
\mathrm{E}\left[\mathbf{1}_{\{g \leq t\}} \varphi\left(I_{\infty}\right)\right]=\mathrm{E}\left[\int_{0}^{t} \varphi\left(I_{S}\right)\left(-\frac{d I_{S}}{I_{S}}\right)\right]=\mathrm{E}\left[\int_{I_{t}}^{r} \varphi(a) \frac{d a}{a}\right] .
$$

Since $\left\{I_{t} \leq a\right\}=\left\{t \geq T_{a}\right\}$ the latter can also be written as

$$
\mathrm{E}\left(\int_{0}^{r} \varphi(a) \frac{d a}{a} \mathbf{1}_{\left\{T_{a} \leq t\right\}}\right)
$$

so that from (9)

$$
\mathrm{E}\left[\mathbf{1}_{\{g \leq t\}} \varphi\left(I_{\infty}\right)\right]=\mathrm{E}\left(\int_{0}^{r} \varphi(a) \frac{d a}{a} \mathbf{1}_{\left\{T_{a} \leq t\right\}}\right) .
$$

Now note that the identity (10) still holds if we replace $t$ by a generic stopping time. Applying again the monotone class theorem gives us then

$$
\mathrm{E}\left[\Phi_{g} \varphi\left(I_{\infty}\right)\right]=\mathrm{E}\left[\int_{0}^{r} \varphi(a) \frac{d a}{a} \Phi_{T_{a}} \mathbf{1}_{\left\{T_{a}<\infty\right\}}\right] .
$$

Finally, using $I_{\infty} \stackrel{\mathcal{L}}{=} r U$ with $U$ being uniform on $[0,1]$ under $P_{r}$ (see $\left.(2)\right)$, we see that identity (11) implies identity (8.1).

Remarks. The statement of the theorem invites for a proof choosing between, on the one hand, initial enlargement with $I_{\infty}$, and, on the other hand, progressive enlargement with $g$. However we have not exactly proceeded like this; the Proposition plays the role of the initial enlargement method and relies on a classical Girsanov relationship between $P_{r}$ and $P_{r}^{\prime}$.

In conclusion we find it interesting to present the above as an example of the potential of enlargement techniques, and here specifically, of a melange of enlargement techniques and Girsanov's theorem. Having said so, we know that this approach can in principle be done for higher dimensions; however, the corresponding Theorem 1 would look more complicated.

For inverse local time properties linking a Bessel-process with an OrnsteinUhlenbeck process see e.g. Hirsch and Yor (2013), Section 2. 


\section{More general context of last-hitting times}

Now, as announced in the Introduction, a brief look at examples outside of mathematical finance.

Buying and selling problems, best-choice problems, secretary problems and others are typical representatives of a last-hitting time problem. One may see them as problems of stopping on the last improvement of a stochastic process. In some of these problems, the difficulty stemming from the last-hitting time character disappears. To give a very simple example, suppose we observe sequentially variables $X_{1}, X_{2}, \cdots$ and would like to maximize, for a given objective function $f$, the expected total return, that is we seek

$$
\arg \max _{\tau} f\left(X_{1}, X_{2}, \cdots, X_{\tau}\right)
$$

Suppose now that the optimal payoff for stopping after time $t$ does not depend on $\mathcal{F}_{t}$, where $\left(\mathcal{F}_{s}\right)$ denotes the natural filtration. Then

$$
\left.\sup _{\tau \geq t} \mathrm{E}\left(f\left(X_{1}, X_{2}, \cdots, X_{\tau}\right) \mid \mathcal{F}_{t}\right)\right)=\sup _{\tau \geq t} \mathrm{E}\left(f\left(X_{t+1}, X_{t+2}, \cdots, X_{\tau}\right)\right)
$$

so that RHS as well as $X_{1}, X_{2}, \cdots, X_{t}$ are both $\mathcal{F}_{t}$-measurable. Hence it suffices to compare at each time $t$ the value $f\left(X_{1}, X_{2}, \cdots, X_{t}\right)$ with the RHS supremum in order to take the optimal decision.

In more difficult problems the $\mathcal{F}_{t}$-independence is typically no longer satisfied. However, external information about the underlying process may help us to change nevertheless the last-hitting time problem into a tractable stopping problem. As examples we may refer to the proof of the 1/e-law of best choice (Bruss (1984)) which changed the sequential problem into a combinatorial problem in a non-sequential setting, or as a more recent example, the solution of the continuous-time last-arrival problem (Bruss and Yor (2012)) where the relevant external information about the underlying process was derived from a related martingale.

There may be many other examples of such a "detour".

And then there are certain other problems where the last hitting time objective is hiding behind other objectives, as for example the objective to discover the first time a random subset of a given set becomes complete. We give only one specific example of this. It is in the important field of clinical trials, more precisely, in the field of so-called compassionate-use clinical trials.

\subsection{Compassionate use clinical trials}

In such trials, a sequence of patients is treated with a drug (sometimes without FDA-approval) which may have serious side effects, the only justification being that it may be as being the last hope for the patients.

Typically, not much is known about the success probability of unapproved drugs or unapproved dosage of (known) medication. This is why compassionate use trials are, as far as possible, set up sequentially so that the physician or 
statistician may learn form preceding observations. We should also mention that such trials may not be permitted in all countries, and that, if they are, they always require a special written consent of patients.

A little reflection shows that such sequential treatments pose a difficult ethical problem. The conscientious physician should try to save all lives which can be saved, and, at the same time, avoid all unnecessary sufferings caused by the sequence of treatments. Since he or she is not a prophet, the goal must be to stop (in a given sequence of patients within a fixed horizon) with maximum probability with the first patient completing the random subset of successes, that is, to stop with the last success. Indeed, then all successes are covered, whereas the remaining patients (de facto not savable by the drug) do not have to suffer unnecessarily. In practice the sequence of treatments should also be stopped (in agreement with the patients) if the current estimate of the success probability drops below a certain bound.

If the success probability for each patient is known beforehand, then the optimal strategy follows immediately from the odds-algorithm (Bruss (2000)); for extensions and newer developments see Ano et al. (2010), Ferguson (2008), Tamaki (2010), and Dendievel (2013)).

If the physician has incomplete information about the respective success probabilities, then the general solution of the optimal stopping problem is an open problem, and as the authors think, an important one. However, interestingly, if the physician has absolutely no information about the success probabilities of the treatment, then the optimal solution is known. It then follows from the notion of stochastic processes with proportional increments introduced in Bruss and Yor (2012), and an additional martingale argument, as Dendievel (2013) has shown in a strongly related problem.

\section{Post scriptum}

As the reader will have noticed, this paper looks unfinished. This is the case and was caused by a very sad event: Professor Marc Yor has left us unexpectedly on January 9, 2014. There may be many people who may have known Marc in person better than the co-author, but I think it is safe to say that everybody who had the pleasure to have known Marc for a longer time, will remember his remarkable profoundness, his scientific generosity, and also warm kindness, and will understand the very deep sadness which fell upon all of us.

Marc and I had worked on this paper in several periods between 2011 and 2013. Following the positive response of interest Francis Hirsch and Marc had received from the Bulletin of the Belgian Math. Society - Simon Stévin for their paper on the Lamperti presentation of a specific inverse local time (see Hirsch and Yor (2013)), Marc suggested that we submit the paper to the same journal. This is what I did on January 13, 2014, sending the paper as it was, that is, as far as it was written by early December 2013 when we had the last phone call exchange on it. Only this Post scriptum is added now.

As the open ends of the preceding text reveal, we had hoped to push certain things further. So for instance, our discussion about the nature of last hitting 
times is still insufficiently connected with the more specific topic of Williams' Decomposition of the BES(3) which is the main part of this paper. This is also true, to some extent, for the discussions of the mentioned applications. However, since we could no longer discuss these points in more detail, I did not feel entitled to write under both our names a corresponding extended version.

Under the given circumstances, Marc and I would be glad to see that this article may serve as a source of inspiration to other readers. A few of our additional discussions should therefore be indicated.

\section{Possible source of inspiration}

Williams (2002) gave an example of a random time $\rho$ associated with Brownian motion such that $\rho$ is not a stopping time but, nevertheless, $\mathrm{E}\left(M_{\rho}\right)=\mathrm{E}\left(M_{0}\right)$ for every uniformly integrable martingale $M$. This result as well as some results of a different kind in Bruss and Ferguson (2002) and in Bruss and Yor (2012), raised our interest. To exemplify the latter, we were surprised to see that a stochastic process which looks like a Poisson process but of which we knew that it cannot be reasonably assumed to be a Poisson process, allows for conclusions precisely as if it were a Poisson process, seemingly being the only candidate for which our conclusion should be true. This coincidence had enabled us to solve the so-called Last-arrival problem which was open, and seen by several colleagues, wrongly so, as an ill-posed problem.

Why is it that in Williams' example the equality $\mathrm{E}\left(M_{\rho}\right)=\mathrm{E}\left(M_{0}\right)$ holds true although $\rho$ is not a stopping time for the martingale $M$ whereas this seems intuitively necessary? Why could Marc and I use the above mentioned Poisson coincidence which allowed to apply the odds-algorithm although independence assumptions make in the original problem no sense at all? Thomas et al. (2007) asked a related question about the odds-algorithm when giving up independence assumptions. More generally, such questions seem to be related to the question why sometimes certain methods work seemingly very well although it is questionable that they can be applied.

Of course, one can always see, a posteriori, the reason why a result is true, but, a priori, this does not mean much. We often discussed whether we fail to see that certain coincidences are not coincidences but rather part of a more general picture. Nobody can hope to get in all generality theorems explaining coincidences, but we felt the fact that several cases involving stopping times and non-stopping times occur in related contexts should draw our attention. Is the notion of a stopping time somewhat too coarse? As we understood, an answer could have a non-negligible impact on questions of how to approach specific applications by more skilful models. (In the context of proportional increments, to which we had intended to return after our paper of 2012, we had also discussed results on self similar processes (Jeanblanc et al. (2002)), on stochastic volatility for Lévy processes (Carr et al. (2003)), and on exponential functionals of Lévy processes (Bertoin and Yor (2005)).

Returning to the main part of this paper, we recall that filtrations, progressive enlargements of filtrations and stopping times were an omnipresent part 
in Marc's way of thinking and expressing his thoughts. This had also a strong influence on his students as well as on so many others. Motivated by Williams's example (Williams (2002)), Nikeghbali and Yor (2005) had introduced so-called pseudo-stopping times, and Williams path decomposition is a special case in this paper. Nikeghbali and Yor (2006) proved then several general path decomposition results taking advantage of the multiplicative decomposition of Azéma's supermartingales for last hitting times as well as pseudo-stopping times connected with the study of Doob's maximal identity and multiplicative decompositions. These were relying in some cases on ideas by Jeulin and others with new results on pseudo-stopping times.

In papers on Doob's maximal identity, it was observed that initially enlarging with a well chosen random variable allows to recover the progressive enlargements formulae by projection. I remember that Marc had mentioned this connection which was now also pointed out by the referee. Moreover the latter saw that in the context of the present paper, this would be the global infimum, and that one may also see formula 9 of the present paper as being intrinsic in the results of Nikeghbali and Platen (2013). This is not mentioned in our paper.

The addition of pseudo-stopping times to the class of optional times seems to refine the classical results on path decompositions in a useful way. It would be great to try to relate all these things together in a more general context. Going beyond this, it would be even nicer if one day one could see the most suitable superset of the class of stopping times, that is, 'broad enough for all practical purposes'. If we think of other domains of Mathematics, we see that, sometimes, such a wish becomes reality.

\section{Acknowledgements}

We like to thank Monique Jeanblanc for providing us with a preprint of Nikeghbali and Platen (2013). Moreover, we gratefully acknowledge financial support of the National Bank of Belgium and of the Alexander von Humboldt-Foundation, respectively.

PS: The first-named author would like to thank the referee for his interesting comments. He firmly believes that Professor Marc Yor would join in thanking.

\section{References}

[1] K. Ano, H. Kakinuma, and N. Miyoshi: Odds theorem with multiple selection chances, J. Appl. Prob., Vol. 47, No 4, 1093-1104 (2010).

[2] Jean Bertoin and Marc Yor: Exponential functionals of Lévy processes, Prob. Surveys, Vol. 2, 191-212, (2005).

[3] F. Thomas Bruss: A unified approach to a class of best-choice problems with an unknown number of options, Annals of Probab., Vol. 12, No 3, 882-889, (1984)

[4] F. Thomas Bruss: Sum the odds to one and stop, Annals of Probab., Vol. 28, No 3, 1384-1391, (2000). 
[5] F. Thomas Bruss and Thomas S. Ferguson: High-risk and competitive investment models, Annals of Appl. Probab. Vol. 12, No 4 , 1202-1226. (2002)

[6] F. Thomas Bruss and Marc Yor: Stochastic processes with proportional increments and the last-arrival problem, Stoch. Proc. and Th. Applic., Vol. 122 (9), 3239-3261, (2012).

[7] P. Carr, H. Geman, DB Madan and M. Yor: Stochastic volatility for Lévy processes, Mathematical Finance, Vol. 13, Issue 3, 345-382, (2003)

[8] Rémi Dendievel: New developments of the odds-theorem, Math. Scientist, Vol. 38, No 2, 111-123 (2013).

[9] Thomas S. Ferguson: The Sum-the-Odds Theorem with Application to a Stopping Game of Sakaguchi, available at http:/ / www.math.ucla.edu/ tom/papers/oddsThm.pdf, pp 1-15, (2008).

[10] Francis Hirsch and Marc Yor: On the remarkable Lamperti presentation of the inverse local time of a radial Ornstein-Uhlenbeck process, Bulletin Belg. Math. Soc. - Simon Stévin, Vol. 20, No 3, 435-449, (2013).

[11] M. Jeanblanc, J. Pitman, M. Yor: Self-similar processes with independent increments associated with Léry and Bessel processes, Stoch. Proc. and Th. Applic., Vol. 100, Issues 1-2, 223-231, (2002).

[12] Th. Jeulin: Semi-martingales et grossissement d'une filtration, Lect. Notes Math. 833, Springer Verlag, (1980).

[13] A. Nikeghbali and E. Platen: A reading guide for last passage times with financial applications in view. Finance and Stochastics, Vol. 17, No 3, 615-640, (2013)

[14] A. Nikeghbali and M. Yor: A definition and some characteristics of pseudostopping times, Annals of Prob., Vol. 33, No 5, 1804-1824. (2005)

[15] A. Nikeghbali and M. Yor: Doob's maximal identity, multiplicative decompositions and enlargements of filtrations, Illinois Journ. Math., Vol. 50, No 1-4, 791-814, (2006).

[16] D. Revuz and M. Yor: Continuous martingales and Brownian motion. 3rd Edition, Springer (1999).

[17] M. Tamaki: Sum the multiplicative odds to one and stop, J. Appl. Prob., Vol. 47, No 3, 761-777 (2010).

[18] E. Thomas, E. Levrat et B. Iung: L'algorithme de Bruss comme contribution une maintenance préventive opportuniste, Sciences et Technologies de l'Automatique, GDR MACS, No 4 (3), 13-18, (2007).

[19] D. Williams: Path decomposition and continuity of local times for onedimensional diffusions, I, Proceed. of London Math. Soc. (3), 28, 738-768, (1974). 
[20] D. Williams: A Non-Stopping Time with the Optional-Stopping Property, Bull. London Math. Soc. 34 , 610-612, (2002).

Université Libre de Bruxelles

Faculté des sciences

Département de Mathématique, Campus Plaine CP 210

B-1050 Brussels, Belgium.

email :tbruss@ulb.ac.be

Université Pierre et Marie Curie

Laboratoire des Probabilités

4, place Jusieu, Tour 56,

F-75252 Paris Cedex 05, France 